\title{
A Canonical UTD Solution for Electromagnetic Scattering by an Electrically Large Impedance Circular Cylinder Illuminated by an Obliquely Incident Plane Wave
}

\author{
Andrés G. Aguilar, Prabhakar H. Pathak,
}

\author{
and Manuel Sierra-Pérez:
}

\begin{abstract}
A uniform geometrical theory of diffraction (UTD) solution is developed for the canonical problem of the electromagnetic (EM) scattering by an electrically large circular cylinder with a uniform impedance boundary condition (IBC), when it is illuminated by an obliquely incident high frequency plane wave. A solution to this canonical problem is first constructed in terms of an exact formulation involving a radially propagating eigenfunction expansion. The latter is converted into a circumferentially propagating eigenfunction expansion suited for large cylinders, via the Watson transform, which is expressed as an integral that is subsequently evaluated asymptotically, for high frequencies, in a uniform manner. The resulting solution is then expressed in the desired UTD ray form. This solution is uniform in the sense that it has the important property that it remains continuous across the transition region on either side of the surface shadow boundary. Outside the shadow boundary transition region it recovers the purely ray optical incident and reflected ray fields on the deep lit side of the shadow boundary and to the modal surface diffracted ray fields on the deep shadow side. The scattered field is seen to have a cross-polarized component due to the coupling between the $T E_{z}$ and $T M_{z}$ waves (where $z$ is the cylinder axis) resulting from the IBC. Such cross-polarization vanishes for normal incidence on the cylinder, and also in the deep lit region for oblique incidence where it properly reduces to the geometrical optics (GO) or ray optical solution. This UTD solution is shown to be very accurate by a numerical comparison with an exact reference solution.
\end{abstract}

Index Terms-Approximation methods, cylinders, uniform theory of diffraction, impedance boundary conditions, scattering, surface waves.

\section{INTRODUCTION}

A uniform geometrical theory of diffraction (UTD) is developed in this paper for analyzing the canonical problem of electromagnetic (EM) scattering by an electrically large circular cylinder with a uniform surface impedance boundary condition (IBC), when it is illuminated by an obliquely incident plane wave field in the high frequency regime. The construction of a UTD solution for this canonical problem is crucial for the development of a corresponding UTD solution for the more general case of diffraction of an arbitrary ray optical field when it is incident on an arbitrary smooth convex surface with a uniform IBC, via the principle of the localization of high frequency fields, as discussed by Pathak et al. in [1] for the convex surface which is a perfect electric conductor (PEC). The subsequent generalization of the canonical asymptotic solution developed here, to treat the scattering by an arbitrary smooth convex surface with an IBC via the UTD, will be discussed in a separate paper because of length considerations.

The canonical UTD solution studied here and its generalization to treat large convex surfaces of non constant curvature, with an IBC, are of current and future interest, for predicting the EM scattering by locally smooth convex parts of highly metallic structures with a material coating, or by a lossy metallic surfaces, as for example in aerospace, maritime and automotive applications. One notes that a thin coating of absorbing material is often used for isolating wave interactions/coupling between a pair of antennas on convex metallic surfaces, or for controlling the scattering by such electrically large locally convex structures (e.g. parts of an aircraft). The above applications provide relevance to a study of the problem such as that considered in this paper. The UTD ray approach is a natural one for dealing with these type of problems in the high frequency regime. Actually, the UTD approach is not only highly efficient but most importantly it provides a vivid physical picture for the mechanisms of scattering and radiation. Indeed, a complex structure can be decomposed into its simpler component scattering events such as edges, smooth convex surfaces, tips, etc. Then, the UTD ray fields, describing the interaction of an arbitrary incident ray optical field with such geometrical features, can be superposed at an observation point to then obtain the desired scattered field from that complex object [2].

The physical picture for the radiation and scattering provided by the UTD ray approach is extremely valuable for engineering design; no other method appears to provide such information directly. It is noted that the UTD is a uniform version of Keller's original geometrical theory of diffraction (GTD) [3], [4]. The GTD was introduced in the 1950s by Keller to systematically describe diffraction, for the first time, in terms of rays more than a century after an initial attempt by Thomas Young. However, the GTD formulation, being purely optical, fails at and near ray optical shadow boundaries where the GTD fields become singular, since the fields cannot remain purely ray optical therein. The UTD removes this singularity and it automatically recovers the purely ray optical behavior of the GTD outside the transition region adjacent to the shadow boundary. The extent of the transition region, where the fields cannot remain purely ray optical, is inversely proportional to the electrical radius of the convex surface at the point of grazing incidence [1]. 
It is noted that there has been some related work reported previously in the literature. In particular, Kim and Wang [5] provided a UTD solution for the EM scattering by a circular cylinder to simulate a thin lossy material coating for the special two dimensional (2-D) situation corresponding to wave illumination normal to the cylinder. Also, Senior and Volakis [6] have reported a similar solution that is valid everywhere sufficiently far away from the surface of a circular cylinder with an IBC for the 2-D case at normal incidence. Both, the previous works in [5], [6], develop their solution via a direct generalization of the work by Pathak [7] for the corresponding $\mathrm{PEC}$ circular cylinder restricted to the case of normal incidence (2-D). Another UTD solution for the scattering by a circular cylinder with an IBC, again in 2-D, was presented in [8]; this solution has a different form than the ones in [5], [6]. Other asymptotic solutions, valid also for three dimensional (3-D) cases, which deal with problems of surface fields excited by sources only when they are placed directly on an IBC circular cylinder are available in [9], [10]. In contrast, the present work is valid for the case of a source not near the cylinder, and is in a form which can be generalized to treat a fully 3-D convex surface with an IBC when it is illuminated by an arbitrary ray optical field incident from an arbitrary direction. Additional papers, which provide some discussion on the nature and validity of the IBC, may be found in [11]-[14].

It is important to note that while a simple relation exists between the UTD solution for normal incidence with that for oblique incidence with a PEC boundary condition, there is no such direct relationship in the IBC case. Hence, it is not possible to provide a simple and direct generalization of the 2-D scattering solution for normal incidence to obtain the corresponding 3-D solution valid for oblique incidence in the case of an IBC circular cylinder. The reason for the latter results from the fact that even though the illumination may be purely $T E_{z}$ or $T M_{z}$ type, the scattered fields are always a combination of both $T E_{z}$ and $T M_{z}$ wave types in the case of oblique incidence for the IBC cylinder. Such coupling of $T E_{z}$ and $T M_{z}$ waves is obviously a consequence of the IBC; in particular, the IBC on the surface couples one wave type to another. Here, $z$ is the axis of the circular cylinder. This coupling creates cross-polarized field components which are not present in the 2-D case.

The paper is organized as follows. Section II describes the exact eigenfunction series solution corresponding to the canonical problem at hand, which is the starting point of the asymptotic UTD solution development. In section III the UTD solution is explicitly developed for the shadow and the lit regions individually. Section IV presents several numerical results for the scattered field for an IBC circular cylinder. Finally, some conclusions are drawn and discussed in section V. An $e^{j \omega t}$ time dependence for the EM fields is assumed and suppressed throughout this paper.

\section{EIGENFUNCTION SOLUTION}

A $T M_{z}$ or $T E_{z}$ polarized EM plane wave is obliquely incident on an infinitely long impedance circular cylinder with an angle $\theta^{i}$, as shown in Fig. 1, and the scattered field is to

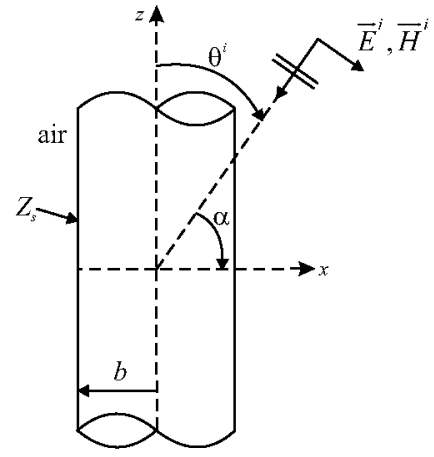

(a) Side view.

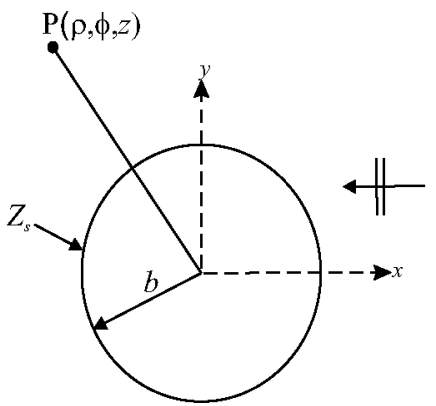

(b) Bottom view.
Fig. 1. Plane wave at oblique incidence on an infinitely long impedance circular cylinder.

be calculated at an observation point $P$. It is assumed that the medium external to the cylinder is free space. The incident field can be described in cylindrical coordinates as

$$
\vec{U}^{i}(\rho, \phi, z)=U_{0}(\hat{\rho} \sin \alpha-\hat{z} \cos \alpha) e^{j k_{0}(z \sin \alpha+\rho \cos \phi \cos \alpha)}
$$

where $k_{0}=\omega \sqrt{\mu_{0} \varepsilon_{0}}$ is the wave number in free space, $U_{0}$ is the field amplitude and $\vec{U}^{i}$ represents either the $\hat{\theta}^{i}$ incident electric or the magnetic field for the $T M_{z}$ or $T E_{z}$ cases, respectively.

One notes that $\hat{\theta}^{i}=\hat{\rho} \sin \alpha-\hat{z} \cos \alpha$. Also, the incident wave arrives on the cylinder along the direction specified by the pair of angles $\left(\theta^{i}, \phi^{i}\right)$, where $\theta^{i}=\frac{\pi}{2}-\alpha$ and $\theta^{i}$ represents the polar angle while $\phi^{i}$ is the azimuthal angle. With no loss of generality, $\phi^{i}=0$ is assumed here for convenience. The $\hat{z}$ components of the incident field are given by

$$
\begin{array}{ll}
E_{z}^{i}=\hat{z} \cdot \vec{U}^{i}, & \text { for the } T M_{z} \text { case; } \\
H_{z}^{i}=\hat{z} \cdot \vec{U}^{i}, & \text { for the } T E_{z} \text { case. }
\end{array}
$$

It is noted that $\vec{H}^{i}$ has no $\hat{z}$ component for the $T M_{z}$ case, while $\vec{E}^{i}$ has no $\hat{z}$ component for the $T E_{z}$ case.

It is useful at this junction to introduce $T M_{z}$ and $T E_{z}$ potentials in terms of which one may express the desired EM fields in a systematic and complete fashion. One notes that a $T M_{z}$ polarized incident field creates a $T M_{z}$ and a $T E_{z}$ polarized scattered field, and likewise, a $T E_{z}$ polarized incident field also creates both, a $T E_{z}$ and $T M_{z}$ scattered field. Both types of field are required to satisfy the IBC. Thus, the wave solution must contain both $T M_{z}$ and $T E_{z}$ field types. Let the potentials $\vec{A}_{z}^{T M}, \vec{F}_{z}^{T M}, \vec{F}_{z}^{T E}$ and $\vec{A}_{z}^{T E}$ be defined as follows [15]:

- For the $T M_{z}$ incident field case: $\vec{A}_{z}^{T M}=\hat{z} A_{z}^{T M}$, $\vec{F}_{z}^{T M}=\hat{z} F_{z}^{T M}$ and

$$
\begin{aligned}
& \vec{H}_{1}=\nabla \times \vec{A}_{z}^{T M}+\frac{1}{j \omega \mu_{0}} \nabla \times \nabla \times \vec{F}_{z}^{T M} \\
& \vec{E}_{1}^{T}=-\nabla \times \vec{F}_{z}^{T M}+\frac{1}{j \omega \varepsilon_{0}} \nabla \times \nabla \times \vec{A}_{z}^{T M}
\end{aligned}
$$


where the $T M_{z}$ type EM fields are produced by $\vec{A}_{z}^{T M}$ and the $T E_{z}$ type EM fields are produced by $\vec{F}_{z}^{T M}$.

- For the $T E_{z}$ incident field case: $\vec{F}_{z}^{T E}=\hat{z} F_{z}^{T E}, \vec{A}_{z}^{T E}=$ $\hat{z} A_{z}^{T E}$ and

$$
\begin{aligned}
& \vec{H}_{2}=\nabla \times \vec{A}_{z}^{T E}+\frac{1}{j \omega \mu_{0}} \nabla \times \nabla \times \vec{F}_{z}^{T E} \\
& \vec{E}_{2}=-\nabla \times \vec{F}_{z}^{T E}+\frac{1}{j \omega \varepsilon_{0}} \nabla \times \nabla \times \vec{A}_{z}^{T E}
\end{aligned}
$$

where the $T E_{z}$ type EM fields are produced by $\vec{F}_{z}^{T E}$ and the $T M_{z}$ type EM fields are produced by $\vec{A}_{z}^{T E}$.

The total EM fields $(\vec{E}, \vec{H})$ are therefore given by

$$
\vec{E}=\vec{E}_{1}+\vec{E}_{2} ; \quad \vec{H}=\vec{H}_{1}+\vec{H}_{2}
$$

One can show that the potentials satisfy the same wave equation as the EM fields [15], namely

$$
\left(\nabla^{2}+k_{0}^{2}\right)\left[\begin{array}{c}
\vec{A}_{z}^{T M} \\
\vec{F}_{z}^{T E}
\end{array}\right]=0
$$

and similarly for $\left(\vec{A}_{z}^{T E} ; \vec{F}_{z}^{T M}\right)$, external to the cylinder (i.e. for $\rho>b$ ). Since the incident plane wave exhibits an $e^{j k_{z} z}$ type behavior with $k_{z}=k_{0} \sin \alpha$, it is clear that all fields and potentials must also satisfy the same $e^{j k_{z} z}$ behavior (this fact will follow directly once the boundary conditions are imposed; here this eventual fact is imposed a priori for convenience). Moreover, the plane wave $e^{j k_{0} \rho \cos \phi \cos \alpha}$ can be represented in terms of cylindrical waves by using the relation [15]

$$
e^{j x \cos \phi}=\sum_{n=-\infty}^{\infty} j^{n} e^{-j n \phi} J_{n}(x)
$$

where $J_{n}(x)$ is the cylindrical Bessel function of order $n$. Thus, one may express the potentials as follows

$$
\left[\begin{array}{c}
\vec{A}_{z} \\
\vec{F}_{z}
\end{array}\right]=e^{j k_{z} z} \hat{z} \sum_{n=-\infty}^{\infty} j^{n} e^{-j n \phi}\left[\begin{array}{c}
\tilde{A}_{z} \\
\tilde{F}_{z}
\end{array}\right]
$$

where

$$
\left(\nabla_{t}^{2}+k_{t 0}^{2}\right)\left[\begin{array}{c}
\tilde{A}_{z}^{T M} \\
\tilde{F}_{z}^{T E}
\end{array}\right]=0
$$

and the transverse wave number $k_{t 0}=\sqrt{k_{0}^{2}-k_{z}^{2}}=k_{0} \cos \alpha$. The potentials must also satisfy appropriate boundary conditions; the latter are determined from the EM boundary conditions where the EM fields are first expressed in terms of potentials. The field scattered by the cylinder satisfies the outgoing wave radiation condition, and the total field satisfies the uniform IBC on the cylinder. The IBC can be expressed as given by Leontovich [16]:

$$
\left[\hat{\rho} \times \vec{E}=Z_{s} \hat{\rho} \times(\hat{\rho} \times \vec{H})\right]_{\mid \rho=b}
$$

where $Z_{s}$ is the uniform surface impedance. This boundary condition can be written in terms of $\vec{E} \cdot \hat{z}=E_{z}$ and $\vec{H} \cdot \hat{z}=H_{z}$, along with

$$
\left[\begin{array}{c}
\vec{E}_{z} \\
\vec{H}_{z}
\end{array}\right]=e^{j k_{z} z} \hat{z} \sum_{n=-\infty}^{\infty} j^{n} e^{-j n \phi}\left[\begin{array}{c}
\tilde{E}_{z} \\
\tilde{H}_{z}
\end{array}\right]
$$

as follows:

$$
\begin{aligned}
& {\left[\frac{\partial \tilde{E}_{z}}{\partial \rho}-j \frac{k_{t 0}^{2}}{k_{0}} \Lambda_{s}^{-1} \tilde{E}_{z}=j \frac{n k_{z}}{k_{0} Y_{0} \rho} \tilde{H}_{z}\right]_{\mid \rho=b}} \\
& {\left[\frac{\partial \tilde{H}_{z}}{\partial \rho}-j \frac{k_{t 0}^{2}}{k_{0}} \Lambda_{s} \tilde{H}_{z}=-j \frac{n k_{z}}{k_{0} Z_{0} \rho} \tilde{E}_{z}\right]_{\mid \rho=b}}
\end{aligned}
$$

where $\Lambda_{s}=\frac{Z_{s}}{Z_{0}}$ is the surface impedance normalized with respect to $Z_{0}=\frac{1}{Y_{0}}=\sqrt{\frac{\mu_{0}}{\varepsilon_{0}}}$, namely to the characteristic impedance in free space. Then, the electric and magnetic vector potentials for $\rho>b$ are expressed in the spectral domain in terms of Hankel functions:

$$
\begin{aligned}
& {\left[\begin{array}{c}
\tilde{A}_{z}^{T M} \\
\tilde{F}_{z}^{T E}
\end{array}\right]=} j \frac{1}{2 k_{t 0}}\left[\begin{array}{c}
Y_{0} \\
Z_{0}
\end{array}\right] \\
& \cdot\left(H_{n}^{(1)}\left(k_{t 0} \rho\right)+\left[\begin{array}{c}
A_{n}^{m} \\
A_{n}^{e}
\end{array}\right] H_{n}^{(2)}\left(k_{t 0} \rho\right)\right) \\
& {\left[\begin{array}{c}
\tilde{F}_{z}^{T M} \\
\tilde{A}_{z}^{T E}
\end{array}\right]=j \frac{1}{k_{t 0}}\left[\begin{array}{c}
C_{n}^{m} \\
C_{n}^{e}
\end{array}\right] H_{n}^{(2)}\left(k_{t 0} \rho\right) }
\end{aligned}
$$

where $n$ is the azimuthal wave number, $k_{z}=k_{0} \sin \alpha$ is the axial wave number and $k_{t 0}=k_{0} \cos \alpha$ is the transverse wave number in free space. In the impedance cylinder problem, as in the dielectric-coated one, the scattered field contains not only fields with the same polarization as the incident wave but also the orthogonal polarization, which is a necessary condition to fulfill the boundary conditions, as stated earlier (i.e. both $T M_{z}$ and $T E_{z}$ polarized fields are present). Thus, vector potentials in (13b) are included to provide the coupled field components with an orthogonal polarization. Superscripts in the vector potentials in (13) indicate the $T M_{z}$ or $T E_{z}$ type of incident field polarization.

By solving the system of equations established by the IBC in (12), the coefficients appearing in (13) are given by

$$
\begin{gathered}
{\left[\begin{array}{c}
A_{n}^{m} \\
A_{n}^{e}
\end{array}\right]=-\frac{\left(\left[\begin{array}{c}
F_{n} N_{n} \\
G_{n} M_{n}
\end{array}\right]+\left(\frac{q_{c}}{m_{t}}\right)^{2} H_{n}^{(2)}\left(k_{t 0} b\right) H_{n}^{(1)}\left(k_{t 0} b\right)\right)}{\left(F_{n} G_{n}+\left(\frac{q_{c}}{m_{t}}\right)^{2}\left(H_{n}^{(2)}\left(k_{t 0} b\right)\right)^{2}\right)}} \\
{\left[\begin{array}{c}
C_{n}^{m} \\
C_{n}^{e}
\end{array}\right] \equiv A_{n}^{c}=\frac{-2 j q_{c}}{\pi m_{t} k_{t 0} b} \frac{1}{\left(F_{n} G_{n}+\left(\frac{q_{c}}{m_{t}}\right)^{2}\left(H_{n}^{(2)}\left(k_{t 0} b\right)\right)^{2}\right)}}
\end{gathered}
$$

where the coupled coefficients $C_{n}^{m, e}$ are independent of the incident field polarization and can be described with one coefficient $A_{n}^{c}$, where $m$ and $e$ superscripts are thus replaced by $c$. Also, 


$$
\begin{aligned}
& {\left[\begin{array}{c}
F_{n} \\
G_{n}
\end{array}\right]=H_{n}^{(2) \prime}\left(k_{t 0} b\right)+\frac{1}{m_{t}}\left[\begin{array}{c}
q_{e} \\
q_{m}
\end{array}\right] H_{n}^{(2)}\left(k_{t 0} b\right)} \\
& {\left[\begin{array}{c}
M_{n} \\
N_{n}
\end{array}\right]=H_{n}^{(1) \prime}\left(k_{t 0} b\right)+\frac{1}{m_{t}}\left[\begin{array}{c}
q_{e} \\
q_{m}
\end{array}\right] H_{n}^{(1)}\left(k_{t 0} b\right)}
\end{aligned}
$$

with $H_{n}^{(1,2)}(z)$ and $H_{n}^{(1,2) \prime}(z)$ being the cylindrical Hankel functions of first and second kind, respectively, and their first derivatives with respect to their argument. It is noted that

$$
\begin{aligned}
& q_{m}=-j m_{t} \Lambda_{s}^{-1} \cos \alpha \\
& q_{e}=-j m_{t} \Lambda_{s} \cos \alpha \\
& q_{c}\left(n, k_{z}\right)=-j m_{t} \frac{n}{k_{t 0} b} \sin \alpha .
\end{aligned}
$$

The above quantities appear in (14) and (15). And it is also noted that the factor $m_{t}=\left(\frac{k_{t 0} b}{2}\right)^{1 / 3}$.

It is useful to study the following limiting cases. When the incident plane wave strikes the cylinder at normal incidence $\left(\alpha \rightarrow 0^{\circ}\right)$, one reduces to the 2 -D case, yielding

$$
\lim _{\alpha \rightarrow 0^{\circ}} q_{c}\left(n, k_{z}\right)=0 .
$$

Also, it is obvious for normal incidence that:

$$
\begin{aligned}
& \lim _{\alpha \rightarrow 0^{0}} A_{n}^{m}=-\frac{H_{n}^{(1) \prime}\left(k_{t 0} b\right)+\frac{q_{m}}{m_{t}} H_{n}^{(1)}\left(k_{t 0} b\right)}{H_{n}^{(2) \prime}\left(k_{t 0} b\right)+\frac{q_{m}}{m_{t}} H_{n}^{(2)}\left(k_{t 0} b\right)} \\
& \lim _{\alpha \rightarrow 0^{\circ}} A_{n}^{e}=-\frac{H_{n}^{(1) \prime}\left(k_{t 0} b\right)+\frac{q_{e}}{m_{t}} H_{n}^{(1)}\left(k_{t 0} b\right)}{H_{n}^{(2) \prime}\left(k_{t 0} b\right)+\frac{q_{e}}{m_{t}} H_{n}^{(2)}\left(k_{t 0} b\right)} \\
& \lim _{\alpha \rightarrow 0^{0}} A_{n}^{c}=0 .
\end{aligned}
$$

Thus, at normal incidence the orthogonal polarization is not excited. The impedance cylinder formulation also recovers the PEC case when the surface impedance $\Lambda_{s} \rightarrow 0$, and therefore

$$
\begin{aligned}
& \lim _{\Lambda_{s} \rightarrow 0} A_{n}^{m}=-\frac{H_{n}^{(1)}\left(k_{t 0} b\right)}{H_{n}^{(2)}\left(k_{t 0} b\right)} \\
& \lim _{\Lambda_{s} \rightarrow 0} A_{n}^{e}=-\frac{H_{n}^{(1)^{\prime}}\left(k_{t 0} b\right)}{H_{n}^{(2)^{\prime}}\left(k_{t 0} b\right)} .
\end{aligned}
$$

The factors in (16) play an important role in the dependence of the fields on the surface impedance. Thus, $q_{m}$ and $q_{e}$ describes the TM and TE creeping waves, respectively, which in the 3-D case $\left(\alpha \neq 0^{\circ}\right)$ are coupled through the $q_{c}$ factor.

\section{UTD WITH IBC FORMULATION}

By applying the Watson Transformation [17] one transforms the discrete variable $n$ into a continuous variable $\nu$. Thus, (13) becomes in the $\nu$ domain the following:

$$
\begin{aligned}
& {\left[\begin{array}{c}
A_{z}^{T M} \\
F_{z}^{T E}
\end{array}\right]=j \frac{1}{2 k_{t 0}}\left[\begin{array}{c}
Y_{0} \\
Z_{0}
\end{array}\right] e^{j k_{z} z} .} \\
& \quad \cdot\left\{\int_{-\infty}^{\infty} d \nu\left(H_{\nu}^{(1)}\left(k_{t 0} \rho\right)+\left[\begin{array}{c}
A_{\nu}^{m} \\
A_{\nu}^{e}
\end{array}\right] H_{\nu}^{(2)}\left(k_{t 0} \rho\right)\right) .\right. \\
& \left.\cdot\left(\sum_{n=0}^{\infty} e^{-j \nu\left(\phi+2 \pi n-\frac{\pi}{2}\right)}+\sum_{n=0}^{\infty} e^{-j \nu\left(-\phi+2 \pi n-\frac{\pi}{2}\right)}\right)\right\} \\
& {\left[\begin{array}{c}
F_{z}^{T M} \\
A_{z}^{T E}
\end{array}\right]=j \frac{1}{k_{t 0}} e^{j k_{z} z}\left\{\int_{-\infty}^{\infty} d \nu A_{\nu}^{c} H_{\nu}^{(2)}\left(k_{t 0} \rho\right) .\right.} \\
& \left.\left(\sum_{n=0}^{\infty} e^{-j \nu\left(\phi+2 \pi n-\frac{\pi}{2}\right)}-\sum_{n=0}^{\infty} e^{-j \nu\left(-\phi+2 \pi n-\frac{\pi}{2}\right)}\right)\right\}
\end{aligned}
$$

where $n$ above in (20) must not be confused with the azimuthal wave number, introduced in (7) and (8); here it represents the contribution to the scattered field from the diffraction of the $n$-th surface (creeping) ray encirclement around the cylinder.

\section{A. Deep Shadow Region and Creeping Waves}

For observation points in the deep shadow region the integral in (20) can be evaluated via the Cauchy Residue theorem leading to a residue series, which is known to converge rapidly in terms of the creeping wave modes. Thus, for a given value of $n$ encirclements:

$$
\begin{array}{r}
{\left[\begin{array}{c}
A_{z}^{T M} \\
F_{z}^{T E}
\end{array}\right]^{ \pm} \sim \sum_{p=1}^{\infty} j \frac{1}{k_{t 0}}\left[\begin{array}{c}
Y_{0} \\
Z_{0}
\end{array}\right] e^{-j k_{0} t \cos ^{2} \alpha}\left[\begin{array}{c}
D_{p}^{m} \\
D_{p}^{e}
\end{array}\right]} \\
\cdot e^{-j \xi \tau_{p} \frac{e^{-j k_{0} s}}{\sqrt{s}}}
\end{array}
$$

$$
\left[\begin{array}{c}
F_{z}^{T M} \\
A_{z}^{T E}
\end{array}\right]^{ \pm} \sim \sum_{p=1}^{\infty}-j \frac{1}{k_{t 0}} e^{-j k_{0} t \cos ^{2} \alpha} D_{p}^{c} e^{-j \xi \tau_{p}} \frac{e^{-j k_{0} s}}{\sqrt{s}}
$$

corresponding to the residues from the $p$ poles. Here, surface diffracted rays are continuously shed along the forward tangent to the creeping rays which traverse geodesic paths on the cylinder after being launched at the point of grazing incidence. In (21), which represents the potentials for the surface diffracted ray fields, $s$ is the distance from the shedding point $Q_{a, b}$ to the observation point in the shadow region $P_{s}$, as in Fig. 2, and the sign + corresponds to a clockwise creeping or surface ray and a sign - to a counterclockwise surface ray, when looking at a top view of the cylinder. Note that the corresponding \pm signs on $t$ and $\xi$ are not included on the right side only for convenience of notation. The following Fock substitution has been applied [18]:

$$
\nu=k_{t 0} b+m_{t} \tau
$$

before evaluating (20) via the residue theorem. The coefficients $D_{p}^{m, e}$ and $D_{p}^{c}$ are the TM/TE and coupled diffraction coefficients, respectively, defined as 
$D_{p}^{m, e}=-\frac{m_{t}}{\cos \alpha} \sqrt{\frac{2 \pi}{k_{0}}} e^{-j \pi / 4} e^{-j\left(\frac{\tau_{p}}{2 u_{g}}\right)^{2}} \frac{W_{1}\left(\tau_{p}\right)}{W_{2}\left(\tau_{p}\right)} \frac{B_{w}^{m, e}\left(\tau_{p}\right)}{D_{w}\left(\tau_{p}\right)}$

$D_{p}^{c}= \pm j \frac{2 m_{t}}{\cos \alpha} \sqrt{\frac{2 \pi}{k_{0}}} e^{-j \pi / 4} e^{-j\left(\frac{\tau_{p}}{2 u_{g}}\right)^{2}} \frac{1}{\left[W_{2}\left(\tau_{p}\right)\right]^{2}} \frac{q_{c}\left(\tau_{p}\right)}{D_{w}\left(\tau_{p}\right)}$

with the coefficients

$$
\begin{gathered}
B_{w}^{m, e}(\tau)=\left[R_{w_{2}}-q_{e, m}\right]\left[R_{w_{1}}-q_{m, e}\right]+q_{c}^{2}(\tau) \\
\begin{array}{r}
D_{w}(\tau)=R_{w_{2}}^{\prime}\left[\left(R_{w_{2}}-q_{m}\right)+\left(R_{w_{2}}-q_{e}\right)\right]+ \\
+2 q_{c}(\tau) q_{c}^{\prime}(\tau)
\end{array}
\end{gathered}
$$

where

$$
\begin{aligned}
& R_{w_{1,2}}=\frac{W_{1,2}^{\prime}(\tau)}{W_{1,2}(\tau)} \\
& R_{w_{1,2}}^{\prime}=\tau-\left(R_{w_{1,2}}\right)^{2} .
\end{aligned}
$$

One notes that $W_{1,2}(\tau)$ and $W_{1,2}^{\prime}(\tau)$ are Fock-type Airy functions and their derivatives with respect to their argument $\tau$. The Airy differential equation $W_{2}^{\prime \prime}(\tau)-\tau W_{2}(\tau)=0$ has been used to calculate (25b).

The complex propagation factors of each $p$-th creeping wave mode are defined by

$$
e^{-j k_{0} t} e^{-\alpha_{p} t}
$$

with the mode attenuation factor (arising from the shedding) given by

$$
\alpha_{p}=j \frac{m_{t} \cos \alpha}{b} \tau_{p}
$$

The factors $q_{m, e}$ are defined in (16a) and (16b), respectively, and $q_{c}$ and its derivative with respect to $\tau$ are expressed as

$$
\begin{aligned}
& q_{c}(\tau)=-j m_{t}\left(1+\frac{\tau}{2 m_{t}^{2}}\right) \sin \alpha \\
& q_{c}^{\prime}(\tau)=-j \frac{1}{2 m_{t}} \sin \alpha .
\end{aligned}
$$

Moreover, $\tau_{p}$ is the $p$-th root of the transcendental equation

$$
\begin{aligned}
{\left[W_{2}^{\prime}(\tau)-q_{e} W_{2}(\tau)\right]\left[W_{2}^{\prime}(\tau)-\right.} & \left.q_{m} W_{2}(\tau)\right]+ \\
& +q_{c}^{2}(\tau)\left[W_{2}(\tau)\right]^{2}=0
\end{aligned}
$$

which corresponds to the Watson type creeping wave poles and also the surface wave poles on the impedance cylinder. It is noted that only the Watson type creeping wave modes remain if the surface impedance vanishes (corresponding to the PEC case), while the surface wave mode constitutes the type of bound wave which propagates on the impedance surface if the cylinder radius becomes infinitely large or is a planar surface. Due to finite radius of the cylinder, the surface wave mode in the present case also sheds energy along the forward

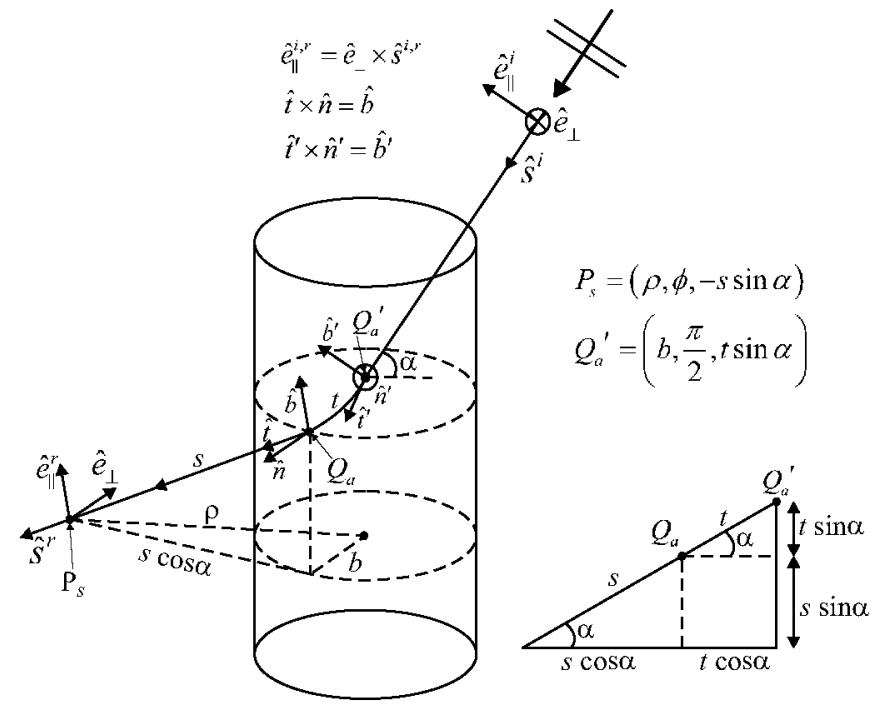

(a) Side view

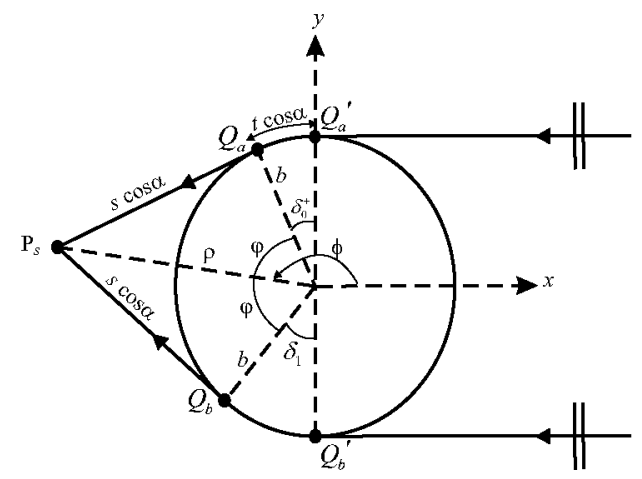

(b) Bottom view. With $\varphi=\cos ^{-1}\left(\frac{b}{\rho}\right)$.

Fig. 2. Ray paths for an observation point in the shadow region.

tangent. The parameter $t=\frac{b \delta_{n}^{ \pm}}{\cos \alpha}$, defined as the length of the surface ray after the $n$-th encirclement around the cylinder, and $\xi=m_{t} \delta_{n}^{ \pm}$, where $\delta_{n}^{ \pm}$is the angle described by $t$, given by

$$
\delta_{n}^{ \pm}=-\left[\cos ^{-1}\left(\frac{b}{\rho}\right)-\left( \pm \phi+2 \pi n-\frac{\pi}{2}\right)\right],
$$

as depicted in Fig. 2(b). Thus, $n=0$ together with the sign + is the first ray field contribution in the shadow region (shortest ray path), and $n=1$ together with the sign - is the second surface ray, while $n=0$ together with the sign - is nonphysically realizable because $\delta_{n}^{ \pm}$must be a positive angle. The exponential term $e^{-j\left(\frac{\tau_{p}}{2 u_{g}}\right)^{2}}$ is a phase correction factor introduced by [8], for improved accuracy when the observation point comes close to the cylinder surface, where

$$
u_{g}=\frac{\cos \alpha}{m_{t}} \sqrt{\frac{k_{0} s}{2}} .
$$

In the shadow region, the fields can be expressed invariantly in terms of a unit vector triad $(\hat{t}, \hat{n}, \hat{b})$ which are fixed in the surface geodesic ray, as is shown in Fig. 2(a). Thus, the scattered field for the $n$-th ray field contribution can be written 
in a compact way in terms of a ray fixed coordinate system as

$$
\vec{E}^{ \pm}\left(P_{s}\right) \sim \vec{E}^{i}\left(Q_{a, b}^{\prime}\right) \cdot \overline{\bar{T}} \frac{e^{-j k_{0} s}}{\sqrt{s}}
$$

where $\vec{E}^{i}\left(Q_{a, b}^{\prime}\right)$ is the incident electric field at the point of grazing on the cylinder, denoted as the launching point $Q_{a, b}^{\prime}$, and $\overline{\bar{T}}$ is a dyadic transfer function which relates the field diffracted from $Q_{a, b}$ to the field incident at $Q_{a, b}^{\prime}$, expressed as

$\overline{\bar{T}}=\sum_{p=1}^{\infty}\left(\hat{b}^{\prime} \hat{b} D_{p}^{m}+\hat{b}^{\prime} \hat{n} D_{p}^{c}+\hat{n}^{\prime} \hat{b} D_{p}^{c}+\hat{n}^{\prime} \hat{n} D_{p}^{e}\right) e^{-\left(\alpha_{p}+j k_{0}\right) t}$

The total scattered field in the shadow region is given by the contributions of all creeping or surface ray contributions. Typically, only the dominant ray paths $n^{+}=0$ and $n^{-}=1$ are retained for sufficiently large radii cylinders; all other multiply encircling rays contribute negligibly in this case.

The location of poles on the complex $\tau$-plane consists in searching for the roots of the transcendental equation in (29), which is of the same form whether a $T M_{z}$ or a $T E_{z}$ incident field is considered. The method proposed starts by calculating the uncoupled zeros of the $T M_{z}$ and $T E_{z}$ modes, upon setting the coupling factor $q_{c}=0$ and by finding separately the roots of

$$
W_{2}^{\prime}(\tau)-q_{m, e} W_{2}(\tau)=0
$$

using as initial guesses the zeros of the corresponding PEC case, which are the zeros of the Airy functions $A i\left(\tau e^{-j 2 \pi / 3}\right)$ and $A i^{\prime}\left(\tau e^{-j 2 \pi / 3}\right)$ for a $T M_{z}$ and a $T E_{z}$ incident field, respectively, tabulated in [19]. Then, it is next possible to find the roots for the transcendental equation in (29), but now with the coupling factor $q_{c} \neq 0$, setting as initial guesses the ones calculated previously. The root finding can be done through Newton-Raphson's method [19]. Zeros of (34) can also be determined numerically by using the procedure in [20], [21], but they may miss one root corresponding to when a surface wave pole is present in addition to the Watson modes. The surface wave mode is often referred to as the Elliot mode in the literature.

\section{B. Shadow Part of Transition Region}

The creeping wave or surface diffracted wave contribution given by the residue (or modal) series in (21) converges rapidly only in the deep shadow. Their convergence is extremely poor as the observer point moves into the transition region around the shadow boundary. For the aforementioned reason, it becomes important to express (20) within the transition region in terms of appropriate expressions with good convergence, as shown next. One begins with the representations in (20) which are exact. Using once more the transformation of (22) again into (20) and introducing the Fock-type Airy functions $W_{1,2}(\tau)$ and $W_{1,2}^{\prime}(\tau)$ as done earlier, one arrives at integral representations that are valid in the shadow part of the transition region. Furthermore, for sufficiently large cylinders these representations also reduce uniformly to the creeping wave modes (via Cauchy residue theorem) as in (21) for the deep shadow region characterized by $\xi \gg 0$. Hence, it is sufficient to use the shadow side transition region expressions given below in (36) even within the deep shadow. In particular, one can easily verify that for an observation point in the shadow part of the transition region, the vector potentials are those in (21) but now with $\overline{\bar{T}}$ replaced by $\overline{\bar{T}}_{U T D}$, where $\overline{\bar{T}}_{U T D}$ is found to be:

$$
\overline{\bar{T}}_{U T D}=\left(\hat{b}^{\prime} \hat{b} D_{m}+\hat{b}^{\prime} \hat{n} D_{c}+\hat{n}^{\prime} \hat{b} D_{c}+\hat{n}^{\prime} \hat{n} D_{e}\right) e^{-j k_{0} t}
$$

where the "uniform" diffraction coefficients, valid everywhere in the shadow region right up to the shadow boundary, are

$$
\begin{aligned}
D_{m, e}= & -\frac{m_{t}}{\cos \alpha} \sqrt{\frac{2}{k_{0}}} e^{-j \pi / 4} . \\
& \cdot\left\{\frac{1}{2 \sqrt{\pi} \xi}\left(1-F\left[k_{t 0} L \tilde{a}\right]\right)+\tilde{P}_{m, e}(\xi, \tau)\right\} \\
D_{c}= & \pm j \frac{m_{t}}{\cos \alpha} \sqrt{\frac{2}{k_{0}}} e^{-j \pi / 4} \tilde{P}_{c}(\xi, \tau)
\end{aligned}
$$

in which $F\left[k_{t 0} L \tilde{a}\right]$ contains a Fresnel integral, as in the transition function of [22], and the geometrical parameters $L=s \cos \alpha$ and $\tilde{a}=\frac{\xi^{2}}{2 m_{t}^{2}}$. The integrals $\tilde{P}_{m, e}(\xi, \tau)$ and $\tilde{P}_{c}(\xi, \tau)$ are the TM/TE and coupled Pekeris functions [7], respectively, given by

$$
\begin{aligned}
& \tilde{P}_{m, e}(\xi, \tau)=\frac{1}{\sqrt{\pi}} \int_{-\infty}^{\infty} d \tau e^{-j \xi \tau} A_{w}^{m, e}(\tau) \\
& \tilde{P}_{c}(\xi, \tau)=\frac{1}{\sqrt{\pi}} \int_{-\infty}^{\infty} d \tau e^{-j \xi \tau} A_{w}^{c}(\tau)
\end{aligned}
$$

with coefficients $A_{w}^{m, e}(\tau)$ and $A_{w}^{c}(\tau)$ expressed in (38), where $V(\tau)$ and $V^{\prime}(\tau)$ is a Fock-type Airy function and its derivative, defined by $2 j V(\tau)=W_{1}(\tau)-W_{2}(\tau)$.

The scattered field everywhere in the shadow region can be written as in (32) with $\overline{\bar{T}}$ therein now replaced by $\overline{\bar{T}}_{U T D}$.

The integration path for the functions $\tilde{P}_{m, e}(\xi, \tau)$ and $\tilde{P}_{c}(\xi, \tau)$ in (37) is deformed where Airy functions decay most rapidly, as is shown in Fig. 3. These Pekeris integrals are solved numerically through a Gauss quadrature, although there are alternative quadrature methods as in [23] or as proposed in $[8]$.

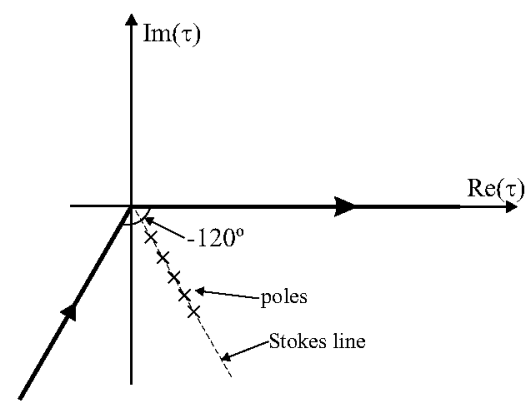

Fig. 3. Integration path for Pekeris integrals $\tilde{P}_{m, e}(\xi, \tau)$ and $\tilde{P}_{c}(\xi, \tau)$ on the complex $\tau$-plane. 


$$
\begin{aligned}
A_{w}^{m, e}(\tau) & =\frac{\left[W_{2}^{\prime}(\tau)-q_{e, m} W_{2}(\tau)\right]\left[V^{\prime}(\tau)-q_{m, e} V(\tau)\right]+q_{c}^{2}(\tau) W_{2}(\tau) V(\tau)}{\left[W_{2}^{\prime}(\tau)-q_{e} W_{2}(\tau)\right]\left[W_{2}^{\prime}(\tau)-q_{m} W_{2}(\tau)\right]+q_{c}^{2}(\tau)\left[W_{2}(\tau)\right]^{2}} \\
A_{w}^{c}(\tau) & =\frac{-j q_{c}(\tau)}{\left[W_{2}^{\prime}(\tau)-q_{e} W_{2}(\tau)\right]\left[W_{2}^{\prime}(\tau)-q_{m} W_{2}(\tau)\right]+q_{c}^{2}(\tau)\left[W_{2}(\tau)\right]^{2}}
\end{aligned}
$$

\section{Lit Part of Transition Region}

For observation points in the lit region, the residue series for the first terms in (20) does not converge. Of course, one must resort to the exact solution of (20) and then evaluate it differently in the lit region. The details are essentially the same as in [7] for the PEC case and are hence not repeated here. For the lit side, one can show that the vector potentials for the direct and reflected incident ray field contributions are

$$
\begin{aligned}
& {\left[\begin{array}{c}
A_{z}^{T M} \\
F_{z}^{T E}
\end{array}\right]=j \frac{1}{k_{t 0}}\left[\begin{array}{c}
Y_{0} \\
Z_{0}
\end{array}\right]\left(e^{j k_{z} z} e^{j k_{t 0} \rho \cos \phi}+\right.} \\
& \left.\quad+e^{j k_{t 0} b \cos \beta}\left[\begin{array}{c}
R_{m} \\
R_{e}
\end{array}\right] \sqrt{\frac{\tilde{\rho}^{\gamma}}{\tilde{\rho}^{\gamma}+l \cos \alpha} e^{-j k_{0} l}}\right) \\
& {\left[\begin{array}{c}
F_{z}^{T M} \\
A_{z}^{T E}
\end{array}\right]=j \frac{1}{k_{t 0}} e^{j k_{t 0} b \cos \beta} R_{c} \sqrt{\frac{\tilde{\rho}^{\gamma}}{\tilde{\rho}^{\gamma}+l \cos \alpha}} e^{-j k_{0} l}}
\end{aligned}
$$

where $l$ is the ray distance from the surface reflected point $Q_{R}$ and the observation point $P_{L}$, and the angle $\beta$ is indicated in Fig. 4(b); $\tilde{\rho}^{\gamma}=\frac{b \cos \beta}{2}$ is the reflected ray caustic distance to the point of reflection; and $R_{m, e}$ and $R_{c}$ are the TM/TE and coupled reflection coefficients, respectively, defined by

$$
\begin{aligned}
R_{m, e} & =-\sqrt{\frac{-4}{\xi^{\prime}}} e^{-j \frac{\xi^{\prime 3}}{12}} e^{-j \pi / 4} . \\
& \cdot\left\{\frac{1}{2 \sqrt{\pi} \xi^{\prime}}\left(1-F\left[k_{t 0} L^{\prime} \tilde{a}^{\prime}\right]\right)+\tilde{P}_{m, e}\left(\xi^{\prime}, \tau\right)\right\} \\
R_{c} & =-\sqrt{\frac{-4}{\xi^{\prime}}} e^{j 2 m_{t}^{2} \xi^{\prime}} e^{-j \pi / 4} \tilde{P}_{c}^{*}\left(\xi^{\prime}, \tau\right)
\end{aligned}
$$

with the parameter $\xi^{\prime}=-2 m_{t} \cos \beta$, and the geometrical parameters $L^{\prime}=l \cos \alpha$ and $\tilde{a}^{\prime}=2 \cos ^{2} \beta$. The first term in (39a) on the right is the incident wave potential; the second term is the potential associated with the reflected wave. The term in (39b) is the coupled or cross-polar component of the reflected wave.

It is of interest to examine (39) for the deep lit region; in particular, one needs to verify if (39) recover the geometrical optics (GO) field description which becomes valid therein. To this end, one first notes that the deep lit region is characterized by $\xi^{\prime} \ll 0$. For the deep lit side, it is observed that the Pekeris function $\tilde{P}_{m, e}\left(\xi^{\prime}, \tau\right)$ is defined as in (37a), and is well behaved for $\xi^{\prime} \ll 0$; but for the coupled Pekeris integral $\tilde{P}_{c}^{*}\left(\xi^{\prime}, \tau\right)$ the function in $(37 \mathrm{~b})$ does not work properly in the deep lit region, because the main contributions there come from the saddle point $\nu_{s}=k_{t 0} b \sin \beta$, which is far from the transition

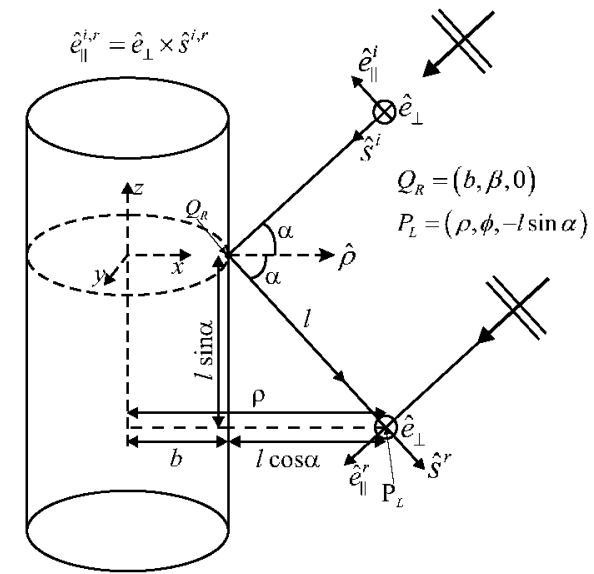

(a) Side view.

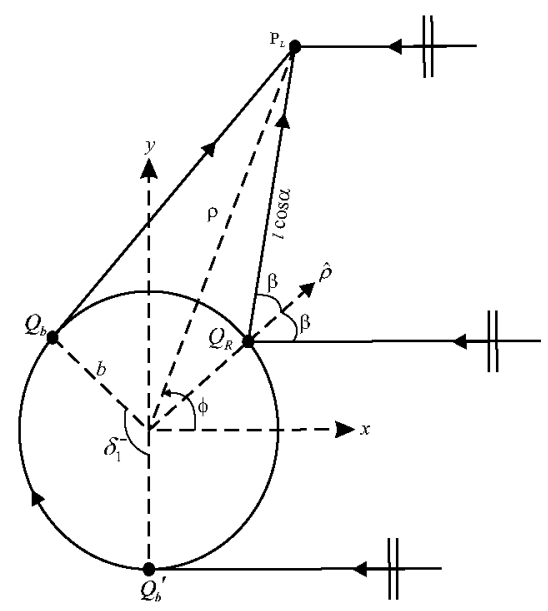

(b) Bottom view.

Fig. 4. Ray paths for an observation point in the lit region.

region, where the Watson's representation of Hankel functions [24] fail. On the other hand, Olver's asymptotic formulas [24] are used here for the Hankel functions in the coefficient $A_{n}^{c}$ in (14b). Moreover, the large argument asymptotic approximation for the Hankel functions [19] when used in (20) also fails, and, therefore, Debye's formulas [25] are applied instead. The integration path is deformed as indicated in Fig. 5, where the path is bent at $\tau_{d}=-2 m_{t}^{2}$, which corresponds with a value $\nu=0$, ensuring that the saddle point is crossed along the integration path and also provides a fast convergence. Thus, $\tilde{P}_{c}^{*}\left(\xi^{\prime}, \tau\right)$ becomes, with these choices, the following:

$\tilde{P}_{c}^{*}\left(\xi^{\prime}, \tau\right)=\frac{1}{\sqrt{\pi}} \int_{-\infty}^{\infty} d \tau e^{-j \frac{\nu^{2}}{2 k_{t 0}(l \cos \alpha+b \cos \beta)}} e^{j \nu(\pi-\phi)} A_{w}^{c^{*}}(\tau)$,

where $\nu$ is related to $\tau$ through the Fock substitution in (22), 


$$
A_{w}^{c^{*}}(\tau)=\frac{-j q_{c}(\tau) \kappa_{1}^{2}}{\left.\left[W_{2}^{\prime}\left(\kappa_{2}\right)-\kappa_{1}^{2} q_{e} W_{2}\left(\kappa_{2}\right)\right]\left[W_{2}^{\prime}\left(\kappa_{2}\right)-\kappa_{1}^{2} q_{m} W_{2}\left(\kappa_{2}\right)\right)\right]+\kappa_{1}^{4} q_{c}^{2}(\tau)\left[W_{2}\left(\kappa_{2}\right)\right]^{2}}
$$

and $A_{w}^{c^{*}}(\tau)$ is expressed in (42), where

$$
\begin{aligned}
\kappa_{1}(\tau) & =\frac{m_{t}}{\nu^{1 / 3}} \gamma(\tau) \\
\kappa_{2}(\tau) & =\nu^{2 / 3} \zeta(\tau),
\end{aligned}
$$

with functions $\zeta(\tau)$ and $\gamma(\tau)$ defined as in [24]. Further approximations to the Fock-type function in (41) are not directly possible.

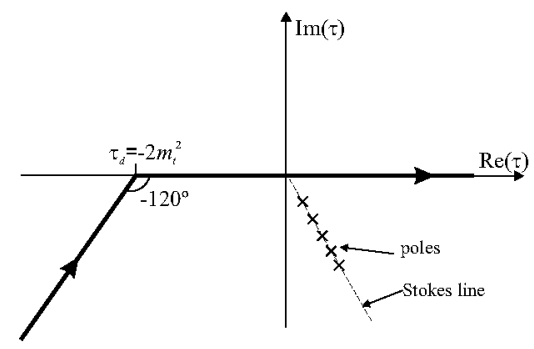

Fig. 5. Integration path for Pekeris integral $\tilde{P}_{c}^{*}\left(\xi^{\prime}, \tau\right)$ on the complex $\tau$ plane.

Then, the scattered field in the lit region can be written in a compact way in terms a ray fixed coordinate system, as indicated in Fig. 4(a), as given by

$$
\vec{E}\left(P_{L}\right) \sim \vec{E}^{i}\left(P_{L}\right)+\vec{E}^{i}\left(Q_{R}\right) \cdot \overline{\bar{R}} \sqrt{\frac{\tilde{\rho}^{\gamma}}{\tilde{\rho}^{\gamma}+l \cos \alpha}} e^{-j k_{0} l}
$$

where $\vec{E}^{i}\left(P_{L}\right)$ is the incident field at an observation point $P_{L}$ and $\vec{E}^{i}\left(Q_{R}\right)$ is the incident field on the surface reflected point $Q_{R}$, and $\overline{\bar{R}}$ is the dyadic reflection function which defines the reflection at $Q_{R}$, namely:

$$
\overline{\bar{R}}=\hat{e}_{\|}^{i} \hat{e}_{\|}^{r} R_{m}+\hat{e}_{\perp} \hat{e}_{\|}^{r} R_{c}+\hat{e}_{\|}^{i} \hat{e}_{\perp} R_{c}+\hat{e}_{\perp} \hat{e}_{\perp} R_{e} .
$$

The unit vector $\hat{e}_{\perp}$ is perpendicular to the plane of incidence, whereas the unit vectors $\hat{e}_{\|}^{i}$ and $\hat{e}_{\|}^{r}$ are in the plane of incidence, as shown in Fig. 4(a). Finally, it is seen that in the deep lit region $\left(\xi^{\prime} \ll 0\right), \overline{\bar{R}} \rightarrow \hat{e}_{\|}^{i} \hat{e}_{\|}^{r} \boldsymbol{R}_{m}+\hat{e}_{\perp} \hat{e}_{\perp} \boldsymbol{R}_{\varepsilon}$ (i.e. $R_{c} \rightarrow 0$ ), where $R_{m, e}$ reduce uniformly to the GO reflection at a locally planar boundary with IBC. For numerical calculation it is thus recommended to use (44) in the deep lit region and in the lit part of the transition region all the way to the shadow boundary. It is thus important to note that (41) should be used instead of (37b).

\section{NUMERICAL RESULTS AND DISCUSSION}

To assess the accuracy of the UTD-based asymptotic solution some numerical results are given. Fig. 6-9 show the scattered field on an IBC circular cylinder. The UTD solution is compared with the corresponding exact eigenfunction series solution and the non uniform GTD solution. Note that the
GTD solution is that which employs (23) for the deep shadow, and (46) for the deep lit region as discussed above. The GTD is not valid in the transition region around the shadow boundary. The UTD solution is valid even within the shadow boundary transition region. A very good agreement is met and, as expected, UTD remains valid within the transition region, where GTD fails. Some differences are seen for the coupled field $\left(E_{\perp}\right.$ and $E_{\|}$for a $T M_{z}$ and a $T E_{z}$ incident field polarization, respectively) at very low field level.

In the shadow region, at least six poles (or six creeping wave modes) are required for the series of (33) to converge. For the IBC cylinder, the creeping wave poles (which pertain to Watson modes) are tightly confined close to the Stokes line and surface wave poles (which pertain to Elliot modes) appear close to the real axis [26]. The Stokes line defines a boundary because when it is crossed the asymptotic behavior of the Hankel function changes, and the Stokes line is located in the fourth quadrant of the complex $\tau$-plane with an approximate angle of $-60^{\circ}$ from the real $\tau$ axis. The creeping wave poles are highly attenuated along the azimuthal direction, while surface waves experience a lower attenuation rate along $\phi$ direction and appear only if the surface impedance is inductive [27]. The presence of the Elliot mode may mean the necessity to add multiple encirclements around the cylinder [8], but in the IBC cylinder examples shown in this section only the first and second ray modes are enough regardless the incident field polarization. These rays are drawn in Fig. 2. In the lit region, besides the incident and reflected ray field contributions, the second creeping ray mode around the cylinder is included, as seen in Fig. 4.

Fig. 10 shows a comparison between the GTD and UTD reflection coefficients as a function of the variable $\xi^{\prime}$ for an impedance circular cylinder. It is observed that far from the transition region UTD recovers the GTD solution accurately. Fig. 10(c) depicts the coupled reflection coefficient and how the coupled Pekeris function $\tilde{P}_{c}(\xi, \tau)$ in $(37 \mathrm{~b})$ works in the transition region but does not recover properly the GTD reflection coefficient in the deep lit region. Nevertheless, the improved coupled Pekeris integral $\tilde{P}_{c}^{+}(\xi, \tau)$ in (41) behaves very well whether in the transition region or in the deep lit region. Furthermore, it is shown that the coupled reflection coefficient is zero at $\phi=0^{\circ}$, as expected.

The aforementioned GTD reflection coefficients are expressed as

$$
\begin{aligned}
& R_{m, e}=-\frac{\left(j m_{t} \cos \beta-q_{e, m}\right)\left(-j m_{t} \cos \beta-q_{m, e}\right)+q_{c}^{2}\left(\nu_{s}\right)}{\left(j m_{t} \cos \beta-q_{e}\right)\left(j m_{t} \cos \beta-q_{m}\right)+q_{c}^{2}\left(\nu_{s}\right)} \\
& R_{c}=\frac{-2 j m_{t} q_{c}\left(\nu_{s}\right) \cos \beta}{\left(j m_{t} \cos \beta-q_{e}\right)\left(j m_{t} \cos \beta-q_{m}\right)+q_{c}^{2}\left(\nu_{s}\right)}
\end{aligned}
$$

where $\nu_{s}=k_{t 0} b \sin \beta$ is the saddle point. 


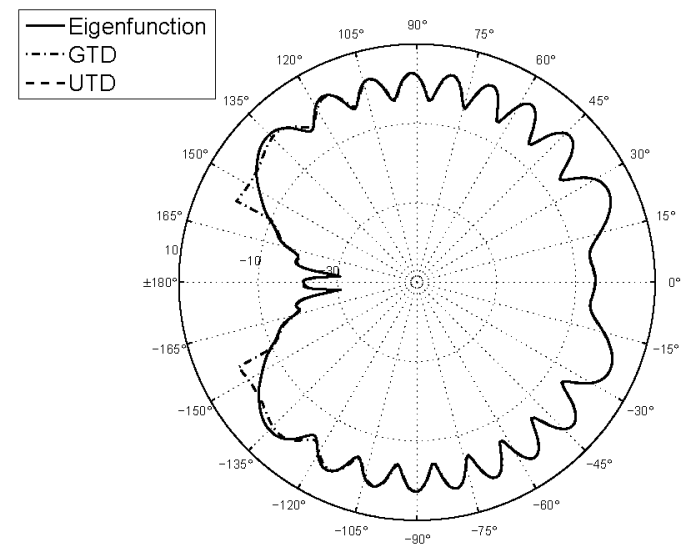

(a) $20 \log \left(\left|E_{\|}\right|\right)(d B)$.

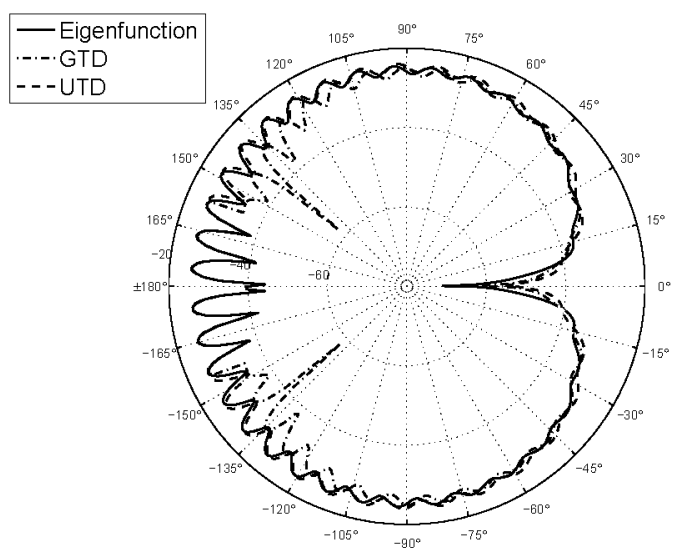

(b) $20 \log \left(\left|E_{\perp}\right|\right)(d B)$.

Fig. 6. Scattered field as a function of $\phi$, for a $T M_{z}$ incident plane wave on an impedance circular cylinder with $\Lambda_{s}=0.25 j, \alpha=20^{\circ}, k_{0} b=20$ and $k_{0} \rho=50$.

\section{CONCLUSion}

A new UTD-based asymptotic solution for scattering on an impedance circular cylinder illuminated by an obliquely incident EM plane wave is introduced. This UTD solution uniformly reduces to the GTD-based asymptotic solution outside the shadow boundary transition region, as it should. In the limit case when the surface impedance $\Lambda_{s} \rightarrow 0$, for both a $T E_{z}$ and a $T M_{z}$ incident field, the PEC case is recovered. In this three dimensional problem the $T E_{z}$ and $T M_{z}$ fields are both present due to coupling at the IBC cylinder. This coupled scattered field has a lower amplitude than the noncoupled one, and it tends to disappear as the incident plane wave approaches normal incidence $\left(\alpha \rightarrow 0^{\circ}\right)$. In accordance with the last property, it is thus noted that the UTD reflection coefficient for the coupled field component is negligible in the deep lit region.

Multiple encirclements are not needed for large cylinders even though the Elliot mode is present. Thus, first and second surface or creeping ray encirclements are included in the shadow region, and direct and reflected rays along with the second creeping ray contribution are used in the lit region.

To effectively use the formulation presented in this work for

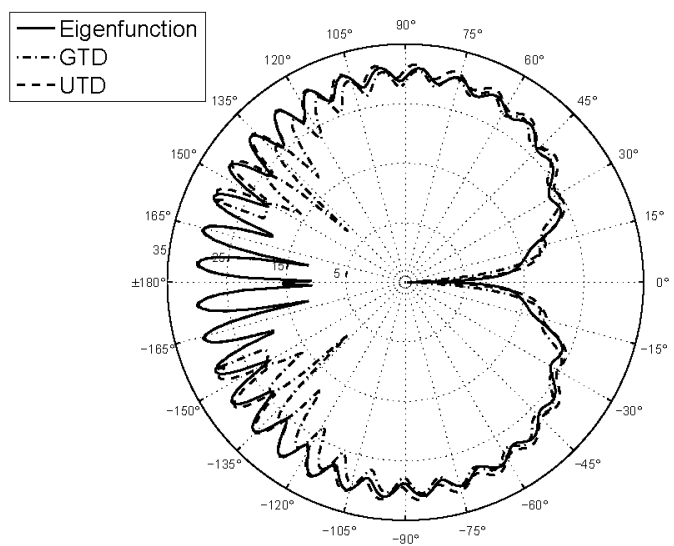

(a) $20 \log \left(\left|E_{||}\right|\right)(d B)$.

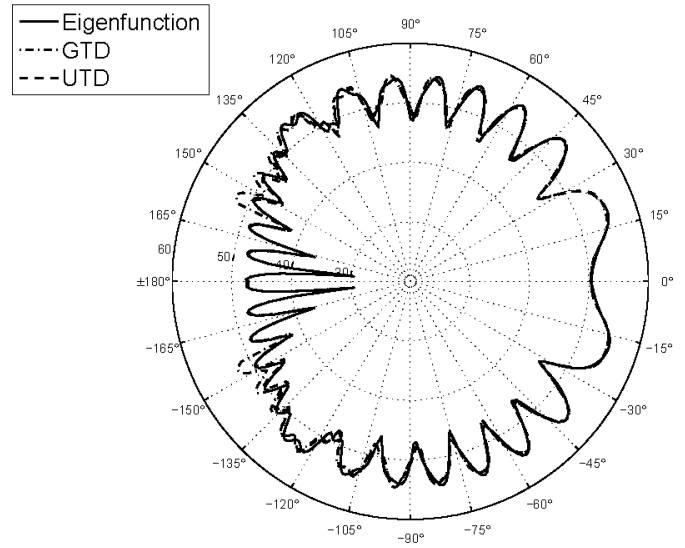

(b) $20 \log \left(\left|E_{\perp}\right|\right)(d B)$.

Fig. 7. Scattered field as a function of $\phi$, for a $T E_{z}$ incident plane wave on an impedance circular cylinder with $\Lambda_{s}=0.25 j, \alpha=30^{\circ}, k_{0} b=20$ and $k_{0} \rho=50$.

dielectric-coated metallic structures, a proper characterization of the surface impedance is needed, where it must depend on the problem geometry and the IBC properties. An effective IBC for thin dielectric coatings will be treated in a separate paper.

The scattering results for the impedance cylinder show a very good agreement between the UTD method and the exact eigenfunction series solution.

\section{APPENDIX}

A. Reduction of the UTD Solution to the Limiting Case of a PEC Circular Cylinder at Normal Incidence

Limiting expressions for the Pekeris integrals in (37) and (41) at normal incidence $\alpha=0^{\circ}$ and for a PEC circular cylinder $\Lambda_{s}=0$ are given by 


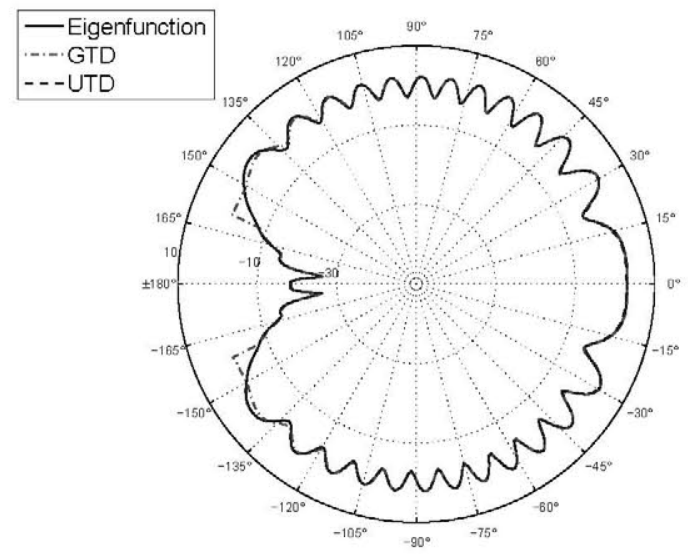

(a) $20 \log \left(\left|E_{\|}\right|\right)(d B)$.

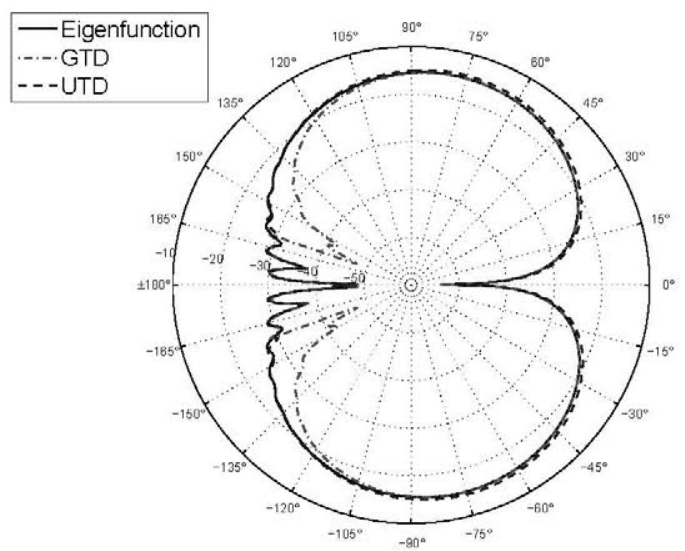

(b) $20 \log \left(\left|E_{\perp}\right|\right)(d B)$

Fig. 8. Scattered field as a function of $\phi$, for a $T M_{z}$ incident plane wave on an impedance circular cylinder with $\Lambda_{s}=1.5 j, \alpha=40^{\circ}, k_{0} b=25$ and $k_{0} \rho=75$.

$$
\begin{aligned}
\lim _{\alpha \rightarrow 0^{0}, \Lambda_{s} \rightarrow 0} \tilde{P}_{m}(\xi, \tau) & =\frac{1}{\sqrt{\pi}} \int_{-\infty}^{\infty} d \tau e^{-j \xi \tau} \frac{V(\tau)}{W_{2}(\tau)} \\
\lim _{\alpha \rightarrow 0^{0}, \Lambda_{s} \rightarrow 0} \tilde{P}_{e}(\xi, \tau) & =\frac{1}{\sqrt{\pi}} \int_{-\infty}^{\infty} d \tau e^{-j \xi \tau} \frac{V^{\prime}(\tau)}{W_{2}^{\prime}(\tau)} \\
\lim _{\alpha \rightarrow 0^{0}, \Lambda_{s} \rightarrow 0} \tilde{P}_{c}(\xi, \tau) & =0 \\
\lim _{\alpha \rightarrow 0^{0}, \Lambda_{s} \rightarrow 0} \tilde{P}_{c}^{*}(\xi, \tau) & =0 .
\end{aligned}
$$

Then, the UTD scattered field given by (32) and (44) as $\alpha \rightarrow 0^{\circ}$ and $\Lambda_{s} \rightarrow 0$ is written as

$$
\begin{aligned}
& \vec{E}^{ \pm}\left(P_{s}\right) \sim-\vec{E}^{i}\left(Q_{a, b}^{\prime}\right) m_{t} \sqrt{\frac{2}{k_{0}}} e^{-j \pi / 4} e^{-j k_{0} t} . \\
& \cdot\left\{\frac{1}{2 \sqrt{\pi} \xi}\left(1-F\left[k_{0} L \tilde{a}\right]\right)+\tilde{P}_{m, e}(\xi, \tau)\right\} \frac{e^{-j k_{0} s}}{\sqrt{s}}
\end{aligned}
$$

in the shadow region, and

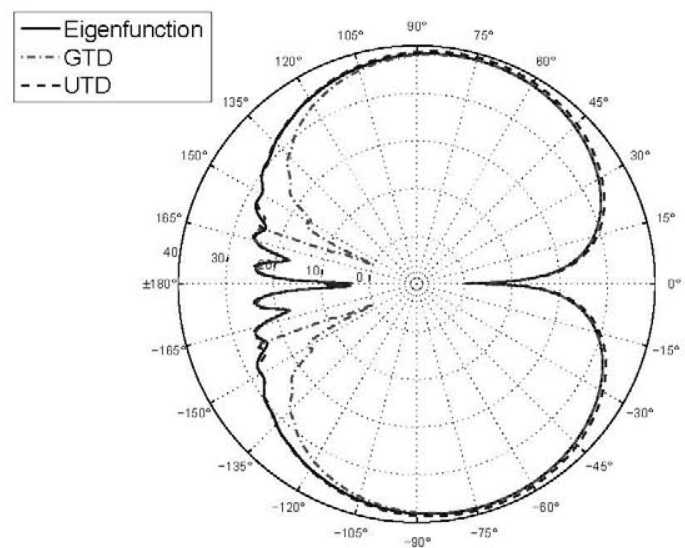

(a) $20 \log \left(\left|E_{\|}\right|\right)(d B)$.

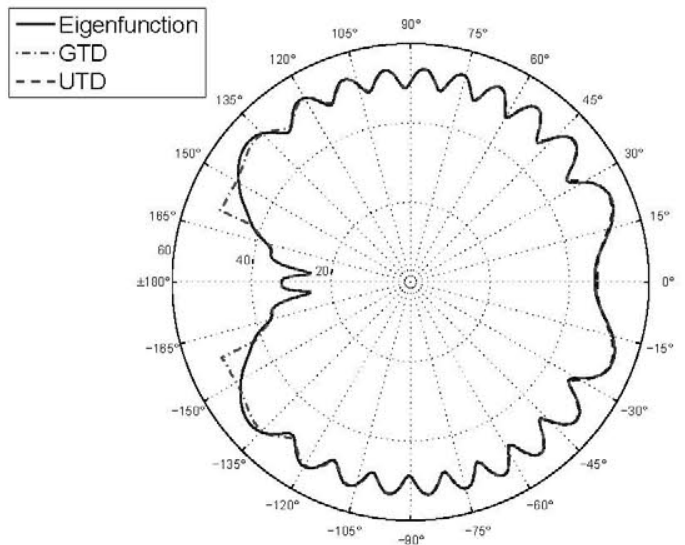

(b) $20 \log \left(\left|E_{\perp}\right|\right)(d B)$.

Fig. 9. Scattered field as a function of $\phi$, for a $T E_{z}$ incident plane wave on an impedance circular cylinder with $\Lambda_{s}=1.5 j, \alpha=50^{\circ}, k_{0} b=25$ and $k_{0} \rho=75$.

$$
\begin{aligned}
& \vec{E}\left(P_{L}\right) \sim \vec{E}^{i}\left(P_{L}\right)-\vec{E}^{i}\left(Q_{R}\right) \sqrt{\frac{-4}{\xi^{\prime}}} e^{-j \frac{\xi^{\prime 3}}{12}} e^{-j \pi / 4} \\
& \cdot\left\{\frac{1}{2 \sqrt{\pi} \xi^{\prime}}\left(1-F\left[k_{0} L^{\prime} \tilde{a}^{\prime}\right]\right)+\tilde{P}_{m, e}\left(\xi^{\prime}, \tau\right)\right\} \sqrt{\frac{\tilde{\rho}^{\gamma}}{\tilde{\rho}^{\gamma}+l}} e^{-j k_{0} l}
\end{aligned}
$$

in the lit region, which is the solution for a PEC circular cylinder at normal incidence given in [7].

\section{ACKNOWLEDGMENT}

The project has the support of the Spanish Ministry of Education, Culture and Sport under reference TEC2011-28789C02-01, a Spanish Ministry of Economy and Competitiveness scholarship (FPI) under reference BES-2009-021462, and the E.T.S.I. Telecomunicacion.

\section{REFERENCES}

[1] P. H. Pathak, W. D. Burnside, and R. J. Marhefka, "A uniform GTD analysis of the diffraction of electromagnetic waves by a smooth convex surface," IEEE Transactions on Antennas and Propagation, vol. 28, no. 5, pp. 631-642, Sep. 1980. 

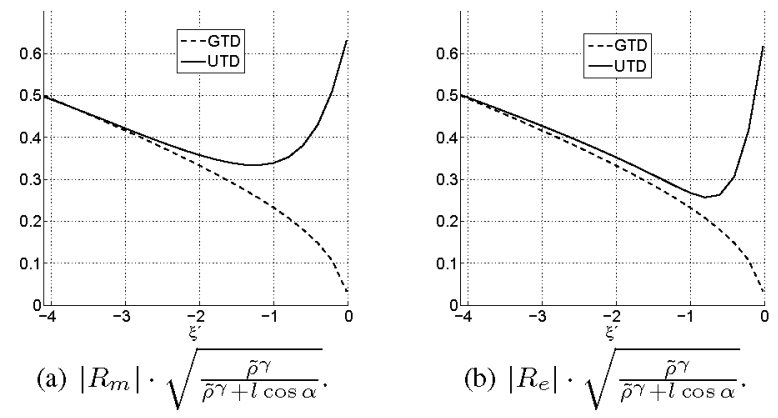

(b) $\left|R_{e}\right| \cdot \sqrt{\frac{\tilde{\rho}^{\gamma}}{\tilde{\rho}^{\gamma}+l \cos \alpha}}$.

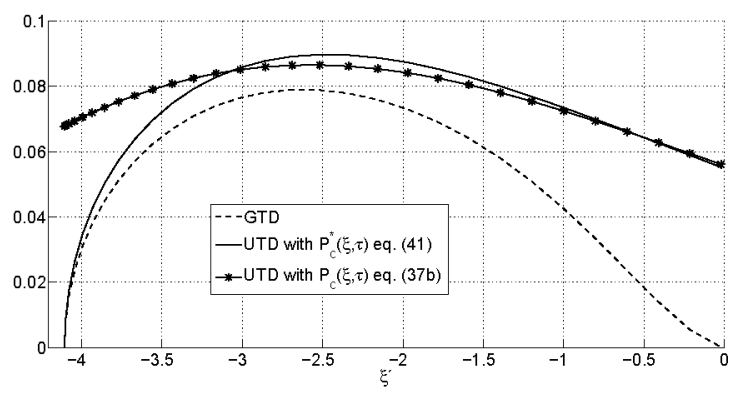

(c) $\left|R_{c}\right| \cdot \sqrt{\frac{\tilde{\rho} \gamma}{\tilde{\rho} \gamma+l \cos \alpha}}$.

Fig. 10. Reflection coefficients for an impedance circular cylinder, for GTD eq. (46) and UTD eq. (40) as a function of $\xi^{\prime}$, with $\Lambda_{s}=0.25 j, \alpha=30^{\circ}$, $k_{0} b=20$ and $k_{0} \rho=50$.

[2] P. H. Pathak, "High frequency techniques for antenna analysis," Proceedings of IEEE, vol. 80, no. 1, pp. 44-65, Jan. 1992.

[3] J. B. Keller, "Geometrical theory of diffraction," Journal of the Optical Society of America, vol. 52, no. 2, pp. 116-130, 1962.

[4] - "Diffraction by a convex cylinder," IEEE Transactions on Antennas and Propagation, vol. 4, no. 2, pp. 312-321, Jul. 1956.

[5] H. T, Kim and N. Wang, "UTD solution for electromagnetic scattering by a circular cylinder with thin lossy coatings," IEEE Transactions on Antennas and Propagation, vol. 37, no. 11, pp. 1463-1472, Nov. 1989.

[6] T. B. A. Senior and J. L. Volakis, Approximate boundary conditions in electromagnetics. The Institution of Electrical Engineers, London, UK, 1995.

[7] P. H. Pathak, "An asymptotic analysis of the scattering of plane waves by a smooth convex cylinder," Radio Science, vol. 14, no. 3, pp. 419-435, May-Jun. 1979.

[8] P. E. Hussar, "A uniform GTD treatment of surface diffraction by impedance and coated cylinders," IEEE Transactions on Antennas and Propagation, vol. 46, no. 7, pp. 998-1008, Jul. 1998.

[9] Ç. Tokgöz and R. J. Marhefka, "A UTD based asymptotic solution for the surface magnetic field on a source excited circular cylinder with an Impedance Boundary Condition," IEEE Transactions on Antennas and Propagation, vol. 54, no. 6, pp. 1750-1757, Jun. 2006.

[10] B. Alisan, V. B. Ertürk, and A. Altintas, "Efficient computation of nonparaxial surface fields excited on a electrically large circular cylinder with an Impedance Boundary Conditions," IEEE Transactions on Antennas and Propagation, vol. 54, no. 9, pp. 2559-2567, Sep. 2006.

[11] S. Kato and N. Wang, "A GTD solution to scattering of plane waves at oblique incidence by a dielectric coated circular cylinder," ElectroScience Laboratory, Dept. Electrical Engineering, Ohio State University, Tech. Rep. 717674-6, Jun. 1988.

[12] K. Steve and N. Wang, "Ray optical formulation for the scattering of an obliquely incident plane wave from an infinitely long dielectric coated circular cylinder," ElectroScience Laboratory, Dept. Electrical Engineering, Ohio State University, Tech. Rep. 717675-5, Jun. 1990.

[13] H. Syed and J. Volakis, "High-frequency scattering by a smooth coated cylinder simulated with generalized impedance boundary conditions." Radio Science, vol. 26, no. 5, pp. 1305-1314, Sep-Oct. 1991.

[14] C. W. Penney, R. J. Luebbers, and J. W. Schuster, "Scattering from coated targets using a frequency-dependent, surface impedance boundary condition in FDTD," IEEE Transactions on Antennas and Propagation, vol. 44, no. 4, pp. 434 443, Apr. 1996.
[15] R. F. Harrington, Time-Harmonic Electromagnetic Fields. John Wiley \& Sons, New York, USA, 2001.

[16] M. A. Leontovich, Investigations on Radiowave Propagation, Part II. Printing House of the Academy of Sciences, Moscow, Russia, 1948.

[17] G. N. Watson, "The diffraction of electric waves by the Earth," Proceedings of the Royal Society of London, vol. 95, pp. 83-99, 1918.

[18] V. A. Fock, "Diffraction of radio waves around the Earth's surface," Journal of Physics USSR, vol. 9, pp. 256-266, 1945.

[19] M. Abramovitz and I. A. Stegun, Handbook of Mathematical Functions. Dover, New York, USA, 1972.

[20] K. P. Spies and J. R. Wait, "On the calculation of the ground wave attenuation factor at low frequencies," IEEE Transactions on Antennas and Propagation, vol. 14, no, 4, pp. 312-321, Jul. 1966.

[21] "On the calculation of antenna patterns for an inhomogeneous spherical Earth," Radio Science, vol. 2, no. 11, pp. 1361-1378, Nov. 1967.

[22] R. G. Kouyoumjian and P. H. Pathak, "A uniform geometrical theory of diffraction for an edge in a perfectly conducting surface," Proc. IEEE vol. 62, no. 11, pp. 1448-1461, Nov. 1974.

[23] L. W. Pearson, "A scheme for automatic computation of Fock-type integrals," IEEE Transactions on Antennas and Propagation, vol. 35 , no. 10 , pp. $1750-1757$, Oct. 1987.

[24] R. Paknys, "Evaluation of Hankel functions with complex argument and complex order," IEEE Transactions on Antennas and Propagation, vol. 40, no. 5, pp. $569-578$, May 1992.

[25] K. Naishadham and L. B. Felsen, "Dispersion of wave guided along a cylindrical substrate-superstrate layered medium," IEEE Transactions on Antennas and Propagation, vol. 41, no. 3, pp. 305-313, Mar. 1993.

[26] R. Paknys and D. R. Jackson, "The relation between creeping waves, leaky waves, and surface waves," IEEE Transactions on Antennas and Propagation, vol. 53, no. 3, pp. 898 - 907, Mar. 2005.

[27] D. A. Hill and J. R. Wait, "Ground wave attenuation function for a spherical Earth with arbitrary surface impedance," Radio Science, vol. 15, no. 3, pp. 637-643, May-Jun. 1980 